\title{
Cytochrome P450 1A2 is the most important enzyme for hepatic metabolism of the metamizole metabolite 4-methylaminoantipyrine
}

\author{
Fabio Bachmann ${ }^{1}$, Urs Duthaler ${ }^{2}$, Henriette Meyerzuschwabidissen ${ }^{3}$, and Stephan \\ Krahenbuhl $^{4}$ \\ ${ }^{1}$ Universitat Basel Departement Biomedizin \\ ${ }^{2}$ University Hospital Basel \\ ${ }^{3}$ University of Basel \\ ${ }^{4}$ University Hospital
}

July 4, 2021

\begin{abstract}
Aim: Metamizole (dipyrone) is a prodrug not detectable in serum or urine after oral ingestion. The primary metabolite is 4methylaminoantipyrine (4-MAA), which can be N-demethylated to 4-aminoantipyrine (4-AA) or oxidized to 4-formylaminoantipyrine (4-FAA) by cytochrome P450 (CYP)-dependent reactions. Our aim was to identify the CYPs involved. Methods: We investigated the metabolism of 4-MAA in vitro using CYP expressing supersomes and the pharmacokinetics of metamizole in the presence of CYP inhibitors in healthy volunteers. Results: The experiments in supersomes revealed CYP1A2 as the major CYP for 4-MAA N-demethylation and 4-FAA formation. CYP2C19 and CYP2D6 contributed to N-demethylation but not to FAA formation. In the subsequent clinical study, we investigated the influence of ciprofloxacin (strong CYP1A2 inhibitor), fluconazole (strong CYP2C19 inhibitor) and the combination ciprofloxacin/fluconazole on the pharmacokinetics of a single dose of metamizole in $\mathrm{n}=12$ healthy volunteers in a randomized, placebo-controlled, double-blind study. Both ciprofloxacin and fluconazole inhibited the metabolism of 4-MAA, confirming the in vitro results. Ciprofloxacin, fluconazole and ciprofloxacin/fluconazole increased the AUC0-12h of 4-MAA by $51 \%, 17 \%$ and $92 \%$, respectively. Ciprofloxacin, fluconazole and ciprofloxacin/fluconazole decreased the AUC0-12h of 4 -AA by $27 \%, 12 \%$ and $24 \%$, respectively, and of 4 -FAA by $33 \%, 9 \%$ and $51 \%$, respectively. Ciprofloxacin, fluconazole and ciprofloxacin/fluconazole increased the half-life of 4-MAA from $3.22 \mathrm{~h}$ (placebo) to $3.91,3.69$ and $6.07 \mathrm{~h}$, respectively. Conclusion: CYP1A2 is the major CYP for the conversion of 4-MAA to 4-AA and 4-FAA. CYP1A2 inhibition increases the 4-MAA exposure by a factor of approximately 1.5, which could be relevant for dose-dependent adverse reactions.
\end{abstract}

Cytochrome P450 1A2 is the most important enzyme for hepatic metabolism of the metamizole metabolite 4-methylaminoantipyrine

Running head: Metabolism of 4-methylaminoantipyrine in humans

Fabio Bachmann ${ }^{1,2,3}$, Henriette E. Meyer zu Schwabedissen ${ }^{4}$, Urs Duthaler ${ }^{1,2,3}$ and Stephan Krähenbühl ${ }^{1,2}$

${ }^{1}$ Division of Clinical Pharmacology \& Toxicology, University Hospital, 4031 Basel, Switzerland

${ }^{2}$ Department of Clinical Research, University of Basel, Switzerland

${ }^{3}$ Department of Biomedicine, University of Basel, Switzerland

${ }^{4}$ Biopharmacy, Department of Pharmaceutical Sciences, University of Basel, Switzerland 
The authors confirm that the Principal Investigator for this paper is Stephan Krähenbühl and that he had direct clinical responsibility for the study participants

Word count (Introduction, Methods, Results and Discussion): 4298

Number of figures and tables: 5 Figures, 1 Table

Key words: metamizole, 4-methylaminantipyrine, 4-formylaminoantipyrine, CYP1A2, CYP2C19

Correspondence

Stephan Krähenbühl, MD, PhD

Clinical Pharmacology \& Toxicology

University Hospital

4031 Basel

Switzerland

e-mail: Stephan.kraehenbuehl@usb.ch

Phone: +4179272 2537

Fax: +4161265 4560

What is already known about this subject:

- Metamizole (dipyrone) is a prodrug not detectable in human body fluids

- Its principal metabolite is 4-methylaminoantipyrine, which is N-demethylated or C-oxidized by CYPdependent reactions

- The CYPs involved in these reactions are currently not known

What this study adds:

- Experiments with supersomes revealed CYP1A2 as the major CYP for both reactions with contributions of CYP2C19 and CYP2D6 to N-demethylation

- These results were confirmed in a clinical study in human healthy volunteers

- Concomitant treatment with strong CYP1A2 inhibitors may be associated with dose-dependent adverse reactions of metamizole

\section{Abstract}

Aim: Metamizole (dipyrone) is a prodrug not detectable in serum or urine after oral ingestion. The primary metabolite is 4-methylaminoantipyrine (4-MAA), which can be N-demethylated to 4-aminoantipyrine (4-AA) or oxidized to 4-formylaminoantipyrine (4-FAA) by cytochrome P450 (CYP)-dependent reactions. Our aim was to identify the CYPs involved.

Methods: We investigated the metabolism of 4-MAA in vitro using CYP expressing supersomes and the pharmacokinetics of metamizole in the presence of CYP inhibitors in healthy volunteers.

Results: The experiments in supersomes revealed CYP1A2 as the major CYP for 4-MAA N-demethylation and 4-FAA formation. CYP2C19 and CYP2D6 contributed to N-demethylation but not to FAA formation. In the subsequent clinical study, we investigated the influence of ciprofloxacin (strong CYP1A2 inhibitor), fluconazole (strong CYP2C19 inhibitor) and the combination ciprofloxacin/fluconazole on the pharmacokinetics of a single dose of metamizole in $n=12$ healthy volunteers in a randomized, placebo-controlled, double-blind study. Both ciprofloxacin and fluconazole inhibited the metabolism of 4-MAA, confirming the in vitro results. Ciprofloxacin, fluconazole and ciprofloxacin/fluconazole increased the $\mathrm{AUC}_{0-12 \mathrm{~h}}$ of 4-MAA by $51 \%, 17 \%$ and $92 \%$, respectively. Ciprofloxacin, fluconazole and ciprofloxacin/fluconazole decreased the 
$\mathrm{AUC}_{0-12 \mathrm{~h}}$ of 4 -AA by $27 \%, 12 \%$ and $24 \%$, respectively, and of 4 -FAA by $33 \%, 9 \%$ and $51 \%$, respectively. Ciprofloxacin, fluconazole and ciprofloxacin/fluconazole increased the half-life of 4-MAA from $3.22 \mathrm{~h}$ (placebo) to $3.91,3.69$ and $6.07 \mathrm{~h}$, respectively.

Conclusion: CYP1A2 is the major CYP for the conversion of 4-MAA to 4-AA and 4-FAA. CYP1A2 inhibition increases the 4-MAA exposure by a factor of approximately 1.5, which could be relevant for dose-dependent adverse reactions.

\section{Introduction}

Metamizole (dipyrone) is an analgesic with antipyretic and spasmolytic properties, which is in use since almost 100 years. Despite many investigations concerning the analgesic effect of metamizole, the analgesic mechanism is currently not completely clear. In a well-designed study, Pierre et al. have shown that the two major metabolites of metamizole, 4-methylaminoantipyrine (4-MAA) and 4-aminoantipyrine (4-AA), inhibit COX-1 and COX-2 by interfering with the $\mathrm{Fe}^{3+}$ atom in the heme of the cyclooxygenases [1]. However, since the typical anti-inflammatory effect of the COX-inhibitors is not observed with metamizole in patients [2], additional, COX-independent mechanisms, may also be involved [3-7]. Similar to the analgesic activity, the spasmolytic effect of metamizole is clinically evident and experimentally established, but the mechanisms are not entirely established. Several possibilities have been proposed, among them opening of ATP sensitive potassium channels [8] and inhibition of G protein-coupled receptor (GPCR) mediated constriction of vascular smooth muscle cells [9].

Metamizole is a prodrug, which is converted to 4-MAA already pre-systemically in the intestinal tract and/or in the liver. 4-MAA has a high oral bioavailability $(>80 \%)$ and is the principal metabolite in plasma [10, 11]. As shown in Figure 1, 4-MAA can be formylated to 4-formylaminoantipyrine (4-FAA) or demethylated to 4-aminoantipyrine (4-AA), which can be acetylated to 4-acetylaminoantipyrine (4-AAA). Less than 5\% of orally administered metamizole is excreted in the urine as 4-MAA, the rest is excreted as 4-AAA, 4-FAA and 4-AA, as well as additional, quantitatively less important metabolites [11-13].

Although the four main metabolites of metamizole have been well-described, only the enzyme responsible for the formation of 4-AAA, a N-acetyltransferase [11, 14, 15] eventually recognized as N-acetyltransferase type 2 (NAT2) [16], has been unequivocally identified. In contrast, the enzymes performing the demethylation and formylation of 4-MAA are so far not known with certainty. Experiments with N,N-dimethyl-4aminoantipyrine (4-DMAA), which carries two instead of one methyl group at the amino position of 4aminoantipyrine, revealed that 4-DMAA can be converted to 4-AA by rat and rabbit liver microsomes, suggesting a cytochrome P450 (CYP)-mediated reaction [17-20]. La Du et al. showed that 4-MAA can be demethylated by isolated rabbit microsomes in a reaction using $\mathrm{NADPH}, \mathrm{Mg}^{2+}$ and oxygen and producing formaldehyde, but this reaction accounted for less than 50\% of 4-MAA degradation [20]. Twenty years after the publication of La Du et al., Noda et al. demonstrated that the oxidative conversion of 4-MAA to 4-FAA accounted for most of the microsomal activity that had not been identified by La Du et al. [21, 22]. In support of these findings, Geisslinger et al. verified that 4-MAA could be converted to 4-AA at a slow rate by human liver microsomes [23]. This reaction could be inhibited by ketoconazole, indicating the involvement of CYP3A4. In addition, patients with impaired liver cirrhosis have a prolonged half-life of 4-MAA, supporting the notion that 4-MAA is metabolized by the liver [15]. In a recent in vitro study, we could confirm that different hepatic CYPs are involved in the N-demethylation of 4-MAA but we also found demethylation activity by myeloperoxidase in neutrophil granulocytes, suggesting that a portion of 4-MAA might be extrahepatically metabolized to 4-AA [24].

Considering the uncertainties regarding N-demethylation of 4-MAA, the aim of the current study was to investigate the metabolism of 4-MAA in vitro using human recombinant CYP isoforms and in humans using established CYP inhibitors. The in vitro experiments were used to identify the most efficient CYPs regarding 4-MAA demethylation, whose contribution was subsequently investigated in vivo. The information 
in humans could also be used to estimate the clinical significance of potential interactions with the CYPs involved in the metabolism of 4-MAA.

\section{Materials and Methods}

\section{Chemicals and reagents}

Dimethylsulfoxide (DMSO), chlorzoxazone, (+)-N-3-benzylnirvanol, ketoconazole, 4-methylpyrazole hydrochloride, quinidine sulphate, sulfaphenazole, ticlopidine hydrochloride, montelukast, 4methylaminoantipyrine hydrochloride (4-MAA), 4-aminoantipyrine (4-AA), 4-acetylaminoantipyrine (4-AAA) and 4-formylaminoantipyrine (4-FAA) were obtained from Sigma-Aldrich (Buchs, Switzerland). Tizanidine hydrochloride, (S)-efavirenz, flurbiprofen, omeprazole, metoprolol, paclitaxel, 6'-hydroxychlorzoxazone, 8'-hydroxyefavirenz, 4'-hydroxyflurbiprofen, $\alpha$-hydroxymetoprolol, 5'hydroxyomeprazole, 6 $\alpha$-hydroxypaclitaxel, hydroxytizanidine, furafylline, ciprofloxacin hydrochloride, fluconazole, chlorzoxazone-d3, efavirenz-d5, flurbiprofen-d3, metoprolol-d6, midazolam-d6, omeprazole-d3, paclitaxel-d5, and tizanidine-d4, 4-MAA-d3, 4-AA-d3, 4-AAA-d3, ciprofloxacin hydrochloride-d8 and fluconazole-d4 were purchased from Toronto Research Chemicals (Toronto, Canada). Midazolam was provided by Roche (Basel, Switzerland) and $\alpha$-Hydroxy-midazolam was acquired from Lipomed (Arlesheim, Switzerland). Corning rhCYP1A2 Supersomes (1 nmol/mL, Lot: 9095001), Corning rhCYP2B6 Supersomes (2 nmol/mL; Lot: 9268001), Corning rhCYP2C8 Supersomes (2 nmol/mL, Lot: 7278001), Corning rhCYP2C9*1 (Arg144) Supersomes (2 nmol/mL, Lot: 9277002), Corning rhCYP2C19 Supersomes (2 nmol/mL, Lot: 9275001), Corning rhCYP2D6*1 Supersomes (1 nmol/mL, Lot: 9274002), Corning rhCYP2E1 Supersomes (2 nmol/mL, lot: 9290001), Corning rhCYP3A4 Supersomes $(2 \mathrm{nmol} / \mathrm{mL}$, Lot: 1006003), NADPH regeneration solution A (26 mM NADP+, $66 \mathrm{mM}$ glucose-6-phosphate, and $66 \mathrm{mM}$ $\mathrm{MgCl} 2$ in $\mathrm{H}_{2} \mathrm{O}$, Lot: 9288003) and $\mathrm{B}(40 \mathrm{U} / \mathrm{mL}$ glucose-6-phosphate dehydrogenase in $5 \mathrm{mM}$ sodium citrate, Lot: 8024003) were obtained from Corning Life Sciences B.V. (Amsterdam, The Netherlands). HPLC grade methanol, HPLC grade water, and formic acid were purchased from Merck (Darmstadt, Germany).

\section{In vitro assays}

4-MAA $(50 \mu \mathrm{M})$ or control substrates (CYP1A2: $10 \mu \mathrm{M}$ tizanidine, CYP2B6: $1 \mu \mathrm{M}$ efavirenz, CYP2C8: $10 \mu \mathrm{M}$ paclitaxel, CYP2C9: $1 \mu \mathrm{M}$ flurbiprofen, CYP2C19: $1 \mu \mathrm{M}$ omeprazole, CYP2D6: $10 \mu \mathrm{M}$ metoprolol, CYP2E1: $1 \mu \mathrm{M}$ chlorzoxazone, CYP3A4: $1 \mu \mathrm{M}$ midazolam) were preincubated for 15 minutes in $100 \mathrm{mM}$ phosphate buffer containing 1.5\% BSA, NADPH-regenerating solution A and B (1:20 dilution and 1:100 dilution, respectively) in the presence or absence of specific CYP inhibitors (CYP1A2: $10 \mu \mathrm{M}$ furaphylline, CYP2B6: $1 \mu \mathrm{M}$ ticlopidine, 2C8: $20 \mu \mathrm{M}$ montelukast, CYP2C9: $10 \mu \mathrm{M}$ sulfaphenazole, CYP2C19: $10 \mu \mathrm{M}$ (1)-N-3-benzylnirvanol, CYP2D6: $1 \mu \mathrm{M}$ quinidine, CYP2E1: $20 \mu \mathrm{M}$ methylpyrazole, CYP3A4: $1 \mu \mathrm{M}$ ketoconazole). The final volume was $500 \mu \mathrm{L}$. The reaction was started by the addition of recombinant supersomes (20 pmol/mL final concentration) and the mixture was incubated on a Thermomixer 5436 (Eppendorf AG, Hamburg, Germany) at $37^{\circ} \mathrm{C}$ and 600 rounds per minute. After 15 minutes, 30 minutes, 1 hour and 2 hours, a sample (CYP substrates: $50 \mu \mathrm{L}, 4$-MAA: $20 \mu \mathrm{L}$ ) was removed and transferred into a autosampler tube containing ice-cold methanol spiked with internal standards (CYP substrates: $150 \mu \mathrm{L}$ methanol containing $25 \mathrm{ng} / \mathrm{mL}$ chlorzoxazone-d3, $50 \mathrm{ng} / \mathrm{mL}$ efavirenz-d5, $50 \mathrm{ng} / \mathrm{mL}$ flurbiprofen-d $3,5 \mathrm{ng} / \mathrm{mL}$ metoprolol-d6, 10 $\mathrm{ng} / \mathrm{mL}$ midazolam-d6, $10 \mathrm{ng} / \mathrm{mL}$ omeprazole-d3, $200 \mathrm{ng} / \mathrm{mL}$ paclitaxel-d5, and $10 \mathrm{ng} / \mathrm{mL}$ tizanidine-d4; 4-MAA: $400 \mu \mathrm{L}$ methanol containing $20 \mathrm{ng} / \mathrm{mL}$ 4-MAA-d3, $30 \mathrm{ng} / \mathrm{mL}$ 4-AA-d3 and $60 \mathrm{ng} / \mathrm{mL}$ 4-AAA-d3). The tubes were vigorously shaken for 30 seconds and stored at $-20^{\circ} \mathrm{C}$ until analysis.

\section{Clinical Study}

We conducted a single center, phase I study (clinicaltrials.gov, ID: NCT04621253) in two successive periods in healthy male Caucasian volunteers. The study was approved by the ethics committee EKNZ (EthikkommissionNordwestschweiz/Zentalschweiz) and Swissmedic and conducted in accordance with good clinical practice guidelines and the current version of the Declaration of Helsinki. The participants were screened 
for any underlying diseases (physical examination, routine laboratory, and electrocardiogram). The use of known CYP inducers (e.g., St. John's Wort) or inhibitors (e.g., grapefruit juice) within 2 weeks before study start was an exclusion criterion as well as excessive caffeine consumption, smoking ( $>5$ cigarettes per day) and use of over-the-counter medication. After signing the informed consent, 12 healthy male subjects were included (mean age: 28.3 years, range 22-39 years, mean body weight: $79.0 \mathrm{~kg}$, range 63-117 kg, mean body mass index: $24.7 \mathrm{~kg} / \mathrm{m}^{2}$, range $21.2-37.3 \mathrm{~kg} / \mathrm{m}^{2}$ ) into the study. A venous blood sample was drawn to determine routine laboratory parameters and the subjects' CYP1A2, CYP2B6, CYP2C9, CYP2C19, and CYP2D6 genotype. The first phase of the study was designed as a randomized, double-blind, 3-arm crossover study. The arms were placebo, ciprofloxacin or fluconazole treatment in a random order. Prior to the study day, the subjects were treated for 3 days with either placebo, ciprofloxacin (750 mg twice daily for 3 days) or fluconazole (400 mg loading dose on day -3 , followed by $200 \mathrm{mg}$ for day -2 and -1 ). Subjects arrived at the study facility in fasted state with $12 \mathrm{~h}$ abstinence of caffeine. The last dose of inhibitor or placebo was taken on the study day (750 mg ciprofloxacin, $200 \mathrm{mg}$ fluconazole or placebo) $1 \mathrm{~h}$ prior to arrival. After arrival in the study facility, a venous catheter was placed in the non-dominant forearm and a blood sample was withdrawn from the catheter to determine the baseline concentrations of the CYP inhibitors. At the same time, participants were treated orally with $1000 \mathrm{mg}$ metamizole (Novalgin ${ }^{\circledR}$ tablets $500 \mathrm{mg}$, Sanofi) and 250 $\mathrm{mL}$ of water. After administration of metamizole, blood samples were drawn after $0.25,0.5,0.75,1,2,3,4 \mathrm{~h}$, $6,8,12$ and 24 hours into EDTA coated tubes. The blood samples were centrifuged at $1500 \mathrm{~g}$ for 10 minutes and the plasma was stored at $-20^{\circ} \mathrm{C}$ until analysis. In the second, open period of the study, 6 randomly chosen participants out of the 12 participants of the first part of the study gave their consent to participate in the study continuation. These 6 participants were treated with the combination of ciprofloxacin and fluconazole with the same schedule as in the first part of the study. The treatment at the study day was the same as described for the first part of study.

To review compliance of the placebo/inhibitor treatment, pill-counting journals were handed out to the subjects. They had to be filled out and returned at the study day for review as well as the empty blisters.

\section{Study Drugs}

The placebo-controlled study medication was produced under GMP conditions by Dr. Hysek Pharmacy, Biel, Switzerland. In short, Ciprofloxacin Helvepharm@ ${ }^{\circledR} 750 \mathrm{mg}$ tablets and Fluconazole Helvepharm ${ }^{\circledR} 200$ mg capsules were over-capsuled and placebo capsules were filled with mannitol. The capsules could not be distinguished. To minimize the risk of potential influence of the treatment order, participants were randomly assigned to one of 6 treatment sequences (A-B-C, A-C-B, B-A-C, B-C-A, C-A-B, C-B-A) by computed randomization. The study staff did not have access to the randomization schedule until the final analysis of the plasma samples.

Novalgin ${ }^{\circledR}$ (500 mg metamizole sodium), Ciprofloxacin Helvepharm ${ }^{\circledR}$ (750 mg ciprofloxacin) and Fluconazole Helvepharm ${ }^{\circledR}$ (200 mg fluconazole) were purchased through the University Hospital Pharmacy Basel, Switzerland.

\section{Genotyping}

The genotypes of CYP1A2, CYP2B6, CYP2C9, CYP2C19 and CYP2D6 were assessed as published previously [25].

\section{Bioanalysis}

All analytes were quantified with high-performance liquid chromatography (Shimadzu, Kyoto, Japan) tandem mass spectrometry (ABSciex, Ontario, Canada). The substrates and the corresponding metabolites (tizanidine, efavirenz, paclitaxel flurbiprofen, omeprazole, metoprolol, chlorzoxazone, midazolam, hydroxytizanidine, 8'-hydroxyefavirenz, 6 $\alpha$-hydroxypaclitaxel, 4'-hydroxyflurbiprofen, 5'-hydroxyomeprazole, $\alpha$ hydroxymetoprolol, 6'-hydroxychlorzoxazone and 1'-hydroxymidazolam) were analyzed according to an earlier publication [26]. The main metabolites of metamizole (4-MAA, 4-AA, 4-FAA, 4-AAA) were quantified with a fully validated method [27]. Quantification of ciprofloxacin and fluconazole was performed by HPLC- 
MS/MS set in positive mode. The following mass transitions were used: ciprofloxacin: $\mathrm{m} / \mathrm{z} 332.3 / 314.2$, ciprofloxacin-d8: m/z 340.3/322.1, fluconazole: $\mathrm{m} / \mathrm{z} 307.1 / 238.0$, fluconazole-d4: $\mathrm{m} / \mathrm{z} 311.2 / 242.1$. As mobile phases, water (mobile phase A and C) and methanol (mobile phase B), both supplemented with 0.1\% formic acid, were applied. Mobile phase $\mathrm{C}$ was added prior to the analytical column using a T-union. The analytes were separated using a core-shell C18 column (Kinetex C18, $2.6 \mu \mathrm{M}, 50 \mathrm{~mm}$ x $2.1 \mathrm{~mm}$, Phenomenex, CA, USA). The following gradient was used (percentage of mobile phase B): 0-0.5 min: 5\%, 0.51-1.6 min: 5\%-60\%, 1.61-2.0 min: 60\%-95\%, 2.01-2.5 min: 95\%, 2.51-3.5 min: 5\%. The initial flow rate for pump A and $\mathrm{B}$ was $0.1 \mathrm{~mL} / \mathrm{min}$. Mobile phase $\mathrm{C}$ was added at an initial rate of $0.5 \mathrm{~mL} / \mathrm{min}$ from the start of the run, decreasing to 0 after 0.5 minutes.

The analysis of the samples met the criteria defined by the US Food and Drug Administration (FDA) guidelines for bioanalytical analysis of study samples [28]. The calibration range was linear from 25-25,000 $\mathrm{ng} / \mathrm{mL}$ for 4-MAA, 25-10,000 ng/mL for 4-AA, 4-AAA and 4-FAA, and 20-10,000 ng/mL for fluconazole and ciprofloxacin. Intra- and interday accuracy between $85.2 \%-114.7 \%$ with an imprecision of less than $13.6 \%$ for all analytes measured.

\section{Pharmacokinetic analysis}

The endpoints for the clinical study was the change in the area under the curve (AUC) of 4-MAA, 4-AA, 4-FAA and 4-AAA and of the terminal half-life $\left(\mathrm{t}_{1 / 2}\right)$ of 4 -MAA under the different conditions (placebo, ciprofloxacin, fluconazole, ciprofloxacin+fluconazole). These parameters were determined using noncompartmental methods with PKanalix (version 2019R1, Lixoft SAS, Abtony, France). AUC $6 \mathrm{~h}, 8 \mathrm{~h}, 12 \mathrm{~h}, 24 \mathrm{~h}$ was assessed using the linear $\log$ trapezoidal method and $t_{1 / 2}$ was calculated from the elimination rate constant, which was determined using linear regression of log concentrations and time.

To describe the influence of the inhibitors on the metabolism of metamizole, metabolic ratios

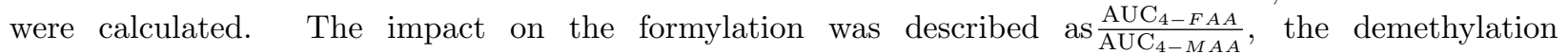

as $\frac{\mathrm{AUC}_{4-A A}+A U C_{4-A A A}}{\mathrm{AUC}_{4-M A A}}$, the acetylation as $\frac{\mathrm{AUC}_{4-A A A}}{\mathrm{AUC}_{4-A A}}$ and the impact on the total metabolism of 4-MAA $\operatorname{as} \frac{\mathrm{AUC}_{4-A A}+A U C_{4-A A A}+A U C_{4-F A A}}{\mathrm{AUC}_{4-M A A}}$.

Both MR and AUCs were assessed at different time points due to the decreasing exposure of the participants to the inhibitors.

\section{Statistics}

MR, AUC and 4-MAA t $t_{1 / 2}$ obtained for the ciprofloxacin, fluconazole, and ciprofloxacin+fluconazole arms were compared to placebo using the Wilcoxon signed rank test. Graphpad Prism (Graphpad Software, La Jolla, California) was used for the statistical analysis. A $P$ value of $<0.05$ was considered as statistically significant $\left({ }^{*} \mathrm{p}<0.05,{ }^{* *} \mathrm{p}<0.01,{ }^{* * *} \mathrm{p}<0.001\right)$.

\section{Results}

In vitro metabolism of 4-MAA

To identify the CYPs responsible for the metabolism of 4-MAA, the principal metabolite of metamizole, we started with investigating the metabolism of 4-MAA in vitro using supersomes. To ensure the functionality of the supersomes used, we first studied the metabolism of the specific CYP substrates in the absence or presence of the corresponding inhibitors. The recombinant CYPs investigated were functional as evidenced by the metabolism of the specific substrates and the formation of the respective metabolites, and the reactions could be blocked or impaired by the addition of the respective inhibitors (suppl. Figure 1). The incubation of 4-MAA with the same supersomes revealed that CYP1A2, CYP2C19 and CYP2D6 formed 4-AA most efficiently (Figure 2). In comparison, CYP2B6, CYP2C9 and CYP2C8 had a measurable but minor 4MAA demethylation activity, while CYP2E1 and CYP3A4 exhibited no detectable activity. Similar to the 
experiments with specific substrates, the formation of 4-AA could be prevented or slowed by the addition of a specific inhibitor. In addition to 4-MAA N-demethylation, the assessment of the formation of 4-FAA showed that the only CYP capable of producing 4-FAA was CYP1A2 (data not shown). To the best of our knowledge, no CYP has so far been identified that catalyzes the formation of 4-FAA from 4-MAA.

\section{Compliance}

Careful review of the pill counting diaries and control of the empty drug blisters indicated that the participants were compliant to the treatment. As displayed in supplementary Figure 2, all participants had residual ciprofloxacin and/or fluconazole plasma concentrations in the morning of the respective study days, also indicating that they were compliant. Surprisingly, when both inhibitors were administered at the same time, the fluconazole plasma concentrations were higher compared to treatment with fluconazole alone, whereas the ciprofloxacin plasma concentrations were in the same range under both conditions.

Effect of ciprofloxacin and fluconazole on the plasma concentrations of 4-MAA, 4-AA, 4-AAA and 4-FAA

Treatment with ciprofloxacin increased the plasma concentrations of 4-MAA (Figure 3) and slowed its elimination (Table 1 and suppl. Figure 3), confirming that 4-MAA is metabolized by CYP1A2. Accordingly, the $\mathrm{AUC}_{0-12 \mathrm{~h}}$ and $\mathrm{AUC}_{0-24 \mathrm{~h}}$ increased by $51 \%$ and $66 \%$, respectively (suppl. Table 1). The addition of fluconazole further slowed the elimination of 4-MAA and increased the $\mathrm{AUC}_{0-12 \mathrm{~h}}$ and $\mathrm{AUC}_{0-24 \mathrm{~h}}$ by $92 \%$ and $133 \%$, respectively, compared to placebo. As expected, the formation of 4-AA, 4-FAA and 4-AAA was slowed and decreased by the administration of ciprofloxacin (Figure 3). The addition of fluconazole further slowed and decreased the formation of 4-FAA and 4-AAA, but not of 4-AA.

Similar to ciprofloxacin, also the treatment with fluconazole increased the plasma concentrations of 4-MAA (Fig. 3) and slowed its elimination (Table 1 and suppl. Figure 3). The increase in the $\mathrm{AUC}_{0-12 \mathrm{~h}}$ and $\mathrm{AUC}_{0-24 \mathrm{~h}}$ was $17 \%$ and $24 \%$, respectively, approximately 5 -times less than the corresponding increase by ciprofloxacin. The addition of ciprofloxacin further slowed the elimination of 4-MAA and increased the $\mathrm{AUC}_{0-12 \mathrm{~h}}$ and $\mathrm{AUC}_{0-24 \mathrm{~h}}$ of 4-MAA as described for ciprofloxacin. Fluconazole retarded and decreased the formation of 4-AA, 4-FAA and 4-AAA slightly with effects on the AUC only up to $8 \mathrm{~h}$ after ingestion of metamizole. The effect of ciprofloxacin, fluconazole and the combination ciprofloxacin/fluconazole on the $\mathrm{AUC}_{0-12 \mathrm{~h}}$ of the four metamizole metabolites is shown in suppl. Fig. 4. The figure shows that the inhibition of the metabolism of 4-MAA is much stronger for ciprofloxacin compared to fluconazole and that the reduction in the $\mathrm{AUC}_{0-12 \mathrm{~h}}$ by ciprofloxacin or fluconazole is more accentuated for 4-FAA than for 4-AA and 4-AAA.

Quantification of the effect of fluconazole and ciprofloxacin on the metabolism of 4-MAA

As shown in Figure 1, the metabolism of 4-MAA is complex. 4-MAA can be converted to 4-AA and 4FAA, and 4-AA can be metabolized further to 4-AAA. Figure 4 shows the change in the AUC of 4-MAA, 4-AA, 4-AA+4-AAA and 4-AA+4-AAA+4-FAA produced by ciprofloxacin, fluconazole and the combination ciprofloxacin/fluconazole at different time points. The effect of fluconazole and ciprofloxacin on the increase in the AUC of 4-MAA grows with time whereas the reduction in the AUC of 4-AA, 4-AAA and 4-FAA by fluconazole and ciprofloxacin decreases with time. Figure 4 visualizes that the effect of fluconazole on the metabolism of 4-MAA is approximately only one third compared to ciprofloxacin. The addition of the AUC of 4-AAA and 4-FAA to the AUC of 4-AA did not change the extent and the pattern of the inhibition of the metabolite formation. The inhibition of the formation of 4-AA and 4-AA+4-AAA+4-FAA by ciprofloxacin or ciprofloxacin/fluconazole was in the range of 50 to $60 \%$ at $6 \mathrm{~h}$ and 30 to $40 \%$ at $12 \mathrm{~h}$ after ingestion of metamizole. The figure also demonstrates that the effects of fluconazole and ciprofloxacin on the increase in the AUC of 4-MAA and on the decrease in the AUCs of 4-AA, 4-FAA and 4-AAA are additive.

An additional possibility to express the inhibition of the metabolism of 4-MAA by ciprofloxacin and fluconazole is by calculating the metabolic ratio, which also considers the increase in the AUC of the substrate (in this case 4-MAA) and not only the decrease in the formation of the metabolites (suppl. Table 2 ). The reduction in the MR was strongest at $6 \mathrm{~h}$ and decreased with time, similar to the effect on the AUC of 4-MAA. The reduction in the MR was more accentuated for ciprofloxacin than fluconazole and 
strongest for the combination ciprofloxacin/fluconazole. The strongest reduction was observed for the couple 4 -FAA/4-MAA at $6 \mathrm{~h}$, reaching $63 \%$ for ciprofloxacin, $24 \%$ for fluconazole and $79 \%$ for the combination ciprofloxacin/fluconazole. At $12 \mathrm{~h}$, the corresponding values were $55 \%, 22 \%$ and $75 \%$ for ciprofloxacin, fluconazole and ciprofloxacin/fluconazole, respectively. In comparison, the effect on the couple 4-AAA/4-AA was much weaker, reaching statistical significance only for ciprofloxacin. This reflects the fact the formation of 4-AAA from 4-AA is dependent on NAT2 and not on CYPs [29].

Effect of the genotype on 4-MAA metabolism

The participants were genotyped for CYP1A2, CYP2B6, CYP2C9, CYP2C19 and CYP2D6 (suppl. Table 3 ). In Figure 5, the (4-AA+4-AAA+4-FAA)/4-MAA MR at $12 \mathrm{~h}$ of the placebo arm is plotted according to the respective genotype of the participants. For CYP2B6 and CYP2D6, there was no visible association between genotype and MR. However, for CYP1A2, 2C9 and 2C19, the MR, which reflects the metabolic activity, correlated with the expected enzymatic activity of the respective genotype.

\section{Discussion}

The aim of the current study was to find out which CYPs are involved in the metabolism of 4-MAA and whether inhibition of the CYPs involved leads to clinically relevant drug interactions. The in vitroexperiments with supersomes revealed that CYP1A2 is the most important enzyme for 4-MAA metabolism and that CYP2C19 and CYP2D6 contribute to 4-MAA N-demethylation. Our in vivo data confirmed these findings and showed that the effects of ciprofloxacin and fluconazole regarding inhibition of the formation of 4-AA and 4-FAA are additive.

Both our in vitro and in vivo experiments showed that CYP1A2 is the dominant enzyme for the demethylation of 4-MAA to 4-AA but also for the conversion of 4-MAA to 4-FAA. So far, it had been demonstrated that both reactions are catalyzed by hepatic microsomes [22-24] but the CYPs involved had not been clearly identified and verified in a clinical study. The results of the current study are in agreement with those in a recent study where we investigated the effect of metamizole on the activity of different CYPs [30]. In this study, we found that metamizole inhibits the conversion of caffeine to paraxanthine, which is catalyzed by CYP1A2, most likely in a competitive fashion. The results of the current study support this interpretation. However, the findings in the current study disagree with the reports of Geisslinger et al. [23] and of Bachmann et al. [24], which were both performed with human microsomes as the enzyme source. Geisslinger et al. showed that the demethylation of 4-MAA to 4-AA by human hepatic microsomes could partially be inhibited by ketoconazole, suggesting a major contribution of CYP3A4 [23]. Geisslinger et al. used a ketoconazole concentration of $10 \mu \mathrm{M}$ in their study, a concentration far below the $\mathrm{K}_{\mathrm{i}}$ of ketoconazole for CYP1A2 and 2C19 [31], excluding inhibition of CYP1A2 and CYPC19 as an explanation for their findings. Bachmann et al. investigated the conversion of 4-MAA to 4-AA by human liver microsomes using specific CYP inhibitors and identified CYP2B6, 2C8, 2C9 and 3A4 as the most important contributors to 4-MAA demethylation [24]. The discrepancies between the current study and the studies of Geisslinger et al. and Bachmann et al. may be due to the different enzyme sources used, human recombinant CYPs expressed in supersomes versus human liver microsomes. The discrepancy is relevant, indicating that results obtained in in vitro studies should be confirmed in another in vitro system or, preferably, by a clinical study. The fact that we obtained almost identical results for the in vitro and in vivo investigations in the current study supports the notion that CYP1A2 is the most important CYP for 4-MAA N-demethylation and conversion to 4-FAA.

The FDA defines strong, moderate and weak enzyme inhibitors as drugs that increase the AUC of a sensitive substrate by [?] 5 -fold, [?] 2 to $<5$-fold, and [?]1.25 to $<2$-fold, respectively. [32]. The FDA lists ciprofloxacin as a strong inhibitor of CYP1A2 and fluconazole as a strong inhibitor of CYP2C19 and a moderate inhibitor of CYP2C9 and CYP3A4. In the current study, ciprofloxacin increased the AUC of 4-MAA time-dependently by a factor of $1.31,1.56$ and 1.51 and fluconazole by a factor of $1.10,1.32$ and 1.17 at 6,8 and $12 \mathrm{~h}$, 
respectively (suppl. Table 1). According to the FDA, the inhibition of 4-MAA metabolism by ciprofloxacin and fluconazole was therefore weak. The reason for an only weak inhibition in the presence of strong inhibitors could be the existence of alternative metabolic pathways. Therefore, we also assessed the combined application of ciprofloxacin and fluconazole, which increased the effect on the AUC of 4-MAA, resulting in AUC ratios of 1.54, 1.90 and 1.92 at 6,8 and $12 \mathrm{~h}$, respectively. The combined application of ciprofloxacin and fluconazole showed that the effects of ciprofloxacin and fluconazole on the AUCs of the metamizole metabolites are additive, excluding a mutual compensation between CYP1A2 and CYP2C19. However, Volz and Kellner have investigated the pharmacokinetics of orally administered ${ }^{14} \mathrm{C}$-labelled metamizole in healthy volunteers [12]. They detected 7 metabolites in serum and could identify four of them as 4-MAA, 4-AA, 4FAA and 4-AAA. Forty-eight hours after administration, $90 \%$ of the radioactivity had been excreted renally, but the four metabolites accounted only for approximately $60 \%$ of the excreted radioactivity. The study therefore indicated the existence of additional metabolic pathways that may compensate for the inhibition of CYP1A2 and CYP2C19.

Based on the results in the supersomes, compensation by CYP2D6 is an obvious possibility, which we didn't study in our clinical trial. However, the activity of CYP2D6 in supersomes was not higher than for CYP2C19, and, in contrast to CYP1A2 and CYP2C19, the CYP2D6 genotype showed no correlation with the metabolic activity, rendering a major contribution of CYP2D6 to the metabolism of 4-MAA unlikely.

An additional possibility is the contribution of myeloperoxidase in granulocytes, as proposed in the report of Bachmann et al. [24]. The results of the current study do not exclude this possibility but suggest that the contribution of this pathway would most likely be less than the contribution by the described hepatic metabolism. If the extrahepatic metabolism were dominant, inhibition of the (in that case minor) hepatic pathway would not be expected to increase the AUC of 4-MAA and to decrease the formation of 4-AA. The inhibition of the (minor) hepatic pathway should be compensated by the dominant extrahepatic pathway. In the current study, pretreatment with ciprofloxacin/fluconazole increased the $\mathrm{AUC}_{0-12 \mathrm{~h}}$ of 4 -MAA by $92 \%$ and decreased the $\mathrm{AUC}_{0-12 \mathrm{~h}}$ of $4-\mathrm{AA}$ by $24 \%$, rendering the existence of a dominant extrahepatic pathway unlikely.

Even if the interaction with CYP1A2 and CYP2C19 inhibitors is quantitatively small, this does not mean that this interaction is clinically negligible. Important adverse reactions of metamizole are hypotensive reactions, skin eruptions, myelotoxicity possibly leading to agranulocytosis and hepatic injury [33]. While hepatic injuries and skin toxicities are mainly dose-independent, immunological reactions [33, 34], hypotensive reactions and myelotoxicity appear to be dose-dependent. Regarding myelotoxicity, a recent genome-wide association study failed to reveal an HLA association and suggested that impaired antioxidative defense mechanisms in granulocytes or granulocyte precursors could be a risk factor for neutropenia and agranulocytosis [35]. In vitro studies using HL60 cells support toxicological mechanisms associated with the formation of reactive metabolites, which is dose-dependent [36,37]. An increase in the plasma concentration of 4-MAA by drug interactions and/or enzyme polymorphisms could therefore enhance the risk for certain adverse reactions associated with metamizole.

In conclusion, we provide evidence that CYP1A2 is the major CYP for the conversion of 4-MAA to 4-AA and 4-FAA. CYP1A2 inhibition increases the 4-MAA exposure by a factor of approximately 1.5, which could be relevant for dose-dependent adverse reactions.

\section{Acknowledgements}

We would like to thank the study nurses Claudia Bläsi and Joyce Jesus de Santos for their valuable help in organizing and performing the study.

Funding : the study was financially supported by the Division of Clinical Pharmacology and Toxicology of the University Hospital of Basel, Switzerland

Conflicts of interest : none of the authors reports any conflict of interest regarding this study 
Data availability : The data that supports the findings of this study are available in the supplementary material of this article.

\section{Literature}

1. Pierre SC, Schmidt R, Brenneis C, Michaelis M, Geisslinger G, Scholich K. Inhibition of cyclooxygenases by dipyrone. British journal of pharmacology 2007; 151: 494-503.

2. Tatsuo MA, Carvalho WM, Silva CV, Miranda AE, Ferreira SH, Francischi JN. Analgesic and antiinflammatory effects of dipyrone in rat adjuvant arthritis model. Inflammation 1994; 18: 399-405.

3. Alves D, Duarte I. Involvement of ATP-sensitive $\mathrm{K}(+)$ channels in the peripheral antinociceptive effect induced by dipyrone. Eur J Pharmacol 2002; 444: 47-52.

4. Escobar W, Ramirez K, Avila C, Limongi R, Vanegas H, Vazquez E. Metamizol, a non-opioid analgesic, acts via endocannabinoids in the PAG-RVM axis during inflammation in rats. European Journal of Pain 2012; 16: 676-89.

5. Ortiz MI, Castaneda-Hernandez G, Granados-Soto V. Possible involvement of potassium channels in peripheral antinociception induced by metamizol: lack of participation of ATP-sensitive $\mathrm{K}+$ channels. Pharmacol Biochem Behav 2003; 74: 465-70.

6. Siebel JS, Beirith A, Calixto JB. Evidence for the involvement of metabotropic glutamatergic, neurokinin 1 receptor pathways and protein kinase $\mathrm{C}$ in the antinociceptive effect of dipyrone in mice. Brain Res 2004; 1003: $61-7$.

7. Vasquez E, Vanegas H. The antinociceptive effect of PAG-microinjected dipyrone in rats is mediated by endogenous opioids of the rostral ventromedical medulla. Brain Res 2000; 854: 249-52.

8. Valenzuela F, García-Saisó S, Lemini C, Ramírez-Solares R, Vidrio H, Mendoza-Fernández V. Metamizol acts as an ATP sensitive potassium channel opener to inhibit the contracting response induced by angiotensin II but not to norepinephrine in rat thoracic aorta smooth muscle. Vascular Pharmacology 2005; 43: 120-27.

9. Gulmez SE, Gurdal H, Tulunay FC. Airway Smooth Muscle Relaxations Induced by Dipyrone. Pharmacology 2006; 78: 202-08.

10. Asmardi G, Jamali F. Pharmacokinetics of dipyrone in man; role of the administration route. Eur J Drug Metab Pharmacokinet 1985; 10: 121-5.

11. Levy M, Zylber-Katz E, Rosenkranz B. Clinical pharmacokinetics of dipyrone and its metabolites. Clin Pharmacokinet 1995; 28: 216-34.

12. Volz M, Kellner HM. Kinetics and metabolism of pyrazolones (propyphenazone, aminopyrine and dipyrone). Br J Clin Pharmacol 1980; 10 Suppl 2: 299S-308S.

13. Zylber-Katz E, Granit L, Levy M. Formation and excretion of dipyrone metabolites in man. Eur J Clin Pharmacol 1992; 42: 187-91.

14. Levy M, Flusser D, Zylber-Katz E, Granit L. Plasma kinetics of dipyrone metabolites in rapid and slow acetylators. Eur J Clin Pharmacol 1984; 27: 453-8.

15. Zylber-Katz E, Caraco Y, Granit L, Levy M. Dipyrone metabolism in liver disease. Clin Pharmacol Ther 1995; 58: 198-209.

16. Agundez JA, Martinez C, Benitez J. Metabolism of aminopyrine and derivatives in man: in vivo study of monomorphic and polymorphic metabolic pathways. Xenobiotica 1995; 25: 417-27.

17. Bast A, Noordhoek J. Product inhibition during the hepatic microsomal N-demethylation of aminopyrine in the rat. Biochem Pharmacol 1981; 30: 19-24. 
18. Imaoka S, Inoue K, Funae Y. Aminopyrine metabolism by multiple forms of cytochrome P-450 from rat liver microsomes: simultaneous quantitation of four aminopyrine metabolites by high-performance liquid chromatography. Arch Biochem Biophys 1988; 265: 159-70.

19. Inoue K, Fujimori K, Mizokami K, Sunouchi M, Takanaka A, Omori Y. Simultaneous determination of aminopyrine hydroxylation and aminopyrine $\mathrm{N}$-demethylation in liver microsomes by high-performance liquid chromatography. J Chromatogr 1983; 274: 201-8.

20. La Du BN, Gaudette L, Trousof N, Brodie BB. Enzymatic dealkylation of aminopyrine (pyramidon) and other alkylamines. J Biol Chem 1955; 214: 741-5.

21. Noda A, Goromaru T, Tsubone N, Matsuyama K, Iguchi S. In vivo formation of 4-formylaminoantipyrine as a new metabolite of aminopyrine. I. Chem Pharm Bull (Tokyo) 1976; 24: 1502-5.

22. Noda A, Tsubone N, Mihara M, Goromaru T, Iguchi S. Formation of 4-formylaminoantipyrine as a new metabolite of aminopyrine. II. Enzymatic demethylation and oxidation of aminopyrine and 4monomethylaminoantipyrine. Chem Pharm Bull (Tokyo) 1976; 24: 3229-31.

23. Geisslinger G, Bocker R, Levy M. High-performance liquid chromatographic analysis of dipyrone metabolites to study their formation in human liver microsomes. Pharm Res 1996; 13: 1272-5.

24. Bachmann F, Duthaler U, Rudin D, Krahenbuhl S, Haschke M. N-demethylation of N-methyl-4aminoantipyrine, the main metabolite of metamizole. Eur J Pharm Sci 2018; 120: 172-80.

25. Bachmann F, Duthaler U, Meyer Zu Schwabedissen HE, Puchkov M, Huwyler J, Haschke M, Krahenbuhl S. Metamizole is a Moderate Cytochrome P450 Inducer Via the Constitutive Androstane Receptor and a Weak Inhibitor of CYP1A2. Clin Pharmacol Ther 2020.

26. Luethi D, Hoener MC, Krahenbuhl S, Liechti ME, Duthaler U. Cytochrome P450 enzymes contribute to the metabolism of LSD to nor-LSD and 2-oxo-3-hydroxy-LSD: Implications for clinical LSD use. Biochem Pharmacol 2019; 164: 129-38.

27. Bachmann F, Blaser L, Haschke M, Krahenbuhl S, Duthaler U. Development and validation of an LCMS/MS method for the bioanalysis of the major metamizole metabolites in human plasma. Bioanalysis 2020; 12: $175-89$.

28. FDA. U.S. Department of Health and Human Services, Food and Drug Andministration (FDA): Bioanalytical Method Validation, Guidance for Industry. In, 2018.

29. Agúndez JA, Carrillo JA, Martínez C, Benítez J. Aminopyrine metabolism in man: the acetylation of aminoantipyrine cosegregates with acetylation of caffeine. Therapeutic drug monitoring 1995; 17: 1-5.

30. Bachmann F, Duthaler U, Meyer Zu Schwabedissen HE, Puchkov M, Huwyler J, Haschke M, Krahenbuhl $\mathrm{S}$. Metamizole is a moderate cytochrome P450 inducer via the constitutive androstane receptor and a weak inhibitor of CYP1A2. Clin Pharmacol Ther 2020: in press.

31. Venkatakrishnan K, von Moltke LL, Greenblatt DJ. Effects of the antifungal agents on oxidative drug metabolism: clinical relevance. Clin Pharmacokinet 2000; 38: 111-80.

32. FDA. Drug Development and Drug Interactions: Table of Substrates, Inhibitors and Inducers. In, 2020.

33. Krisai P, Rudin D, Grünig D, Scherer K, Pichler W, Terracciano L, Krähenbühl S. Acute Liver Failure in a Patient Treated With Metamizole. Front Pharmacol 2019; 10: 996.

34. Blanca-López N, Pérez-Sánchez N, Agúndez JA, García-Martin E, Torres MJ, Cornejo-García JA, Perkins JR, Miranda MA, Andreu I, Mayorga C, Canto G, Blanca M, Doña I. Allergic Reactions to Metamizole: Immediate and Delayed Responses. International archives of allergy and immunology 2016; 169: 223-30.

35. Cismaru AL, Rudin D, Ibañez L, Liakoni E, Bonadies N, Kreutz R, Carvajal A, Lucena MI, Martin J, Sancho Ponce E, Molokhia M, Eriksson N, Eu DACC, Krähenbühl S, Largiadèr CR, Haschke M, Hallberg 
P, Wadelius M, Amstutz U. Genome-Wide Association Study of Metamizole-Induced Agranulocytosis in European Populations. Genes 2020; 11.

36. Rudin D, Lanzilotto A, Bachmann F, Housecroft CE, Constable EC, Drewe J, Haschke M, Krähenbühl S. Non-immunological toxicological mechanisms of metamizole-associated neutropenia in HL60 cells. Biochem Pharmacol 2019; 163: 345-56.

37. Rudin D, Roos NJ, Duthaler U, Krähenbühl S. Toxicity of metamizole on differentiating HL60 cells and human neutrophil granulocytes. Toxicology 2019; 426: 152254.

\section{Legends to Figures}

Figure 1

Metabolism of metamizole. The metamizole is non-enzymatically cleaved to 4-methyl-aminoantipyrine (4MAA) already in the intestine. 4-MAA is the principal metabolite in plasma and can be converted by CYP-dependent reactions to 4-aminoantipyrine (4-AA) or 4-formylaminoantipyrine (4-FAA). 4-AA can be acetylated by N-acetyltransferase 2 (NAT2) to 4-acetyl-aminoantipyrine (4-AAA). The CYPs involved in the metabolism of 4-MAA have not clearly been identified.

Figure 2

In vitro metabolism of 4-methyl-aminoantipyrine (4-MAA). The N-demethylation of 4-MAA to 4aminoantipyrine (4-AA) was investigatedin vitro . The reactions were performed in the absence and presence of the specific inhibitors furaphylline (CYP1A2), ticlopidine (CYP2B6), montelukast (CYP2C8), sulfaphenazole (CYP2C9),(1)-N-3-benzylnirvanol (CYP2C19), quinidine (CYP2D6), methylpyrazole (CYP2E1), and ketoconazole (CYP3A4). The results are displayed mean \pm SEM of 6 independent measurements.

Figure 3

Effect of fluconazole, ciprofloxacin and the combination ciprofloxacin/fluconazole on the plasma concentrations of 4-methyl-aminoantipyrine (4-MAA), 4-aminoantyprine (4-AA), 4-formylaminoantipyrine (4-FAA) and 4-acetyl-aminoantipyrine (4-AAA).Healthy male participants were pretreated with placebo $(\mathrm{n}=12)$, ciprofloxacin ( $\mathrm{n}=12 ; 750 \mathrm{mg}$ twice daily for 3 days), fluconazole $(\mathrm{n}=12,400 \mathrm{mg}$ loading dose on day -3 , followed by $200 \mathrm{mg}$ for day -2 and -1 ) or the combination ciprofloxacin/fluconazole ( $\mathrm{n}=6$; ciprofloxacin and fluconazole as above) before oral administration of $1 \mathrm{~g}$ metamizole. 4-MAA, 4-AA, 4-FAA and 4-AAA plasma concentrations were determined by LC-MS/MS. The results are displayed as mean \pm SEM.

Figure 4

Effect of fluconazole (FLU), ciprofloxacin (CIP) and the combination fluconazole/ciprofloxacin (CIP/FLU) on the area under the curve (AUC) of the metamizole metabolites.AUCs were calculated based on the plasma concentrations shown in Figure 3 using the linear log trapezoidal method. The graph displays the mean \pm SEM of the differences to placebo. $\mathrm{N}=12$ male, healthy participants for placebo, fluconazole and ciprofloxacin, and $\mathrm{n}=6$ for the combination fluconazole/ciprofloxacin. Results are displayed as mean \pm SEM. ${ }^{* * *} \mathrm{p}<0.001$ vs. zero and ${ }^{*} \mathrm{p}<0.05$ vs. zero.

Figure 5

Effect of the CYP genotype on the metabolic ratio (MR) of 4-MAA.The $\mathrm{MR}$ was calculated as $\left(\mathrm{AUC}_{12 \mathrm{~h}}\right.$ 4-AA $+\mathrm{AUC}_{12 \mathrm{~h}}$ 4-AAA $\left.+\mathrm{AUC}_{12 \mathrm{~h} \text { 4-FAA }}\right) / \mathrm{AUC}_{12 \mathrm{~h} \text { 4-MAA }}$ and the results displayed according to the genotype of the different CYPs assessed. An increase in the MR reflects an increase in the activity of a specific CYP. Individual data are plotted.

\section{Table 1}

Pharmacokinetic parameters of the main metabolites of metamizole. Male, healthy were pretreated with placebo, fluconazole, ciprofloxacin and the combination fluconazole/ciprofloxacin before assessing the metabolism of metamizole. Values are given as geometric mean with the $95 \%$ confidence interval in brackets. The 
elimination half-lives of 4-aminoantipyrine, 4-formylaminoantipyrine and 4-acetylaminoantipyrine could not be calculated. ${ }^{* * * *} \mathrm{p}<0.0001,{ }^{* * *} \mathrm{p}<0.001,{ }^{* *} \mathrm{p}<0.01,{ }^{*} \mathrm{p}<0.05$ vs. placebo.

\begin{tabular}{|c|c|c|c|c|}
\hline & Placebo $(n=12)$ & $\begin{array}{l}\text { Fluconazole } \\
(n=12)\end{array}$ & $\begin{array}{l}\text { Ciprofloxacin } \\
(n=12)\end{array}$ & $\begin{array}{l}\text { Fluconazole/Ci- } \\
\text { profloxacin } \\
(n=6)\end{array}$ \\
\hline 4-Methyl- & 4-Methyl- & 4-Methyl- & 4-Methyl- & 4-Methyl- \\
\hline $\begin{array}{l}\text { aminoantipyrine } \\
\text { (4-MAA) }\end{array}$ & $\begin{array}{l}\text { aminoantipyrine } \\
\text { (4-MAA) }\end{array}$ & $\begin{array}{l}\text { aminoantipyrine } \\
\text { (4-MAA) }\end{array}$ & $\begin{array}{l}\text { aminoantipyrine } \\
\text { (4-MAA) }\end{array}$ & $\begin{array}{l}\text { aminoantipyrine } \\
\text { (4-MAA) }\end{array}$ \\
\hline $\mathrm{AUC}_{12 \mathrm{~h}}(\mu \mathrm{g} / \mathrm{L} \mathrm{x}$ & 75,030 & $88,070^{* *}$ & $113,200^{* * *}$ & $144,300^{*}$ \\
\hline & $(65,160-86,390)$ & $(72,910-106,400)$ & $(98,930-129,500)$ & $(124,700-166,900)$ \\
\hline $\mathrm{C}_{\max }(\mathrm{ng} / \mathrm{mL})$ & $\begin{array}{l}15,150 \\
(13,770-16,670)\end{array}$ & $\begin{array}{l}15,380 \\
(13,740-17,220)\end{array}$ & $\begin{array}{l}16,420^{*} \\
(14,820-18,190)\end{array}$ & $\begin{array}{l}17,690 \\
(15,220-20,560)\end{array}$ \\
\hline $\mathrm{T}_{1 / 2}(\mathrm{~h})$ & $3.22(2.84-3.64)$ & $3.69^{*}(3.03-4.49)$ & $3.91^{* * *}(3.43-4.45)$ & $6.07^{*}(5.37-6.86)$ \\
\hline $4-$ & $4-$ & $4-$ & $4-$ & $4-$ \\
\hline $\begin{array}{l}\text { Aminoantipyrine } \\
\text { (4-AA) }\end{array}$ & $\begin{array}{l}\text { Aminoantipyrine } \\
\text { (4-AA) }\end{array}$ & $\begin{array}{l}\text { Aminoantipyrine } \\
\text { (4-AA) }\end{array}$ & $\begin{array}{l}\text { Aminoantipyrine } \\
\text { (4-AA) }\end{array}$ & $\begin{array}{l}\text { Aminoantipyrine } \\
\text { (4-AA) }\end{array}$ \\
\hline $\mathrm{AUC}_{12 \mathrm{~h}}(\mu \mathrm{g} / \mathrm{L} \mathrm{x}$ & 13,680 & $12,110^{*}$ & $9,937^{* * *}$ & $10,350^{*}$ \\
\hline & $(9,730-19,240)$ & $(8,730-16,810)$ & $(7,150-13,810)$ & $(6,717-15,930)$ \\
\hline $\mathrm{C}_{\max }(\mathrm{ng} / \mathrm{mL})$ & $\begin{array}{l}1,621 \\
(1,212-2,167)\end{array}$ & $\begin{array}{l}1,380^{*} \\
(1,043-1,826)\end{array}$ & $\begin{array}{l}1,156^{* * *} \\
(814-1,642)\end{array}$ & $1,178^{*}(737-1,884)$ \\
\hline $4-$ & $4-$ & $4-$ & $4-$ & $4-$ \\
\hline \multicolumn{5}{|c|}{ FormylaminoantipyrFøæmylaminoantipyrFæemylaminoantipyrFøe mylaminoantipyrFøe mylaminoantipyrine } \\
\hline (4-FAA) & $(4-F A A)$ & (4-FAA) & $(4-F A A)$ & (4-FAA) \\
\hline $\mathrm{AUC}_{12 \mathrm{~h}}(\mu \mathrm{g} / \mathrm{L} \mathrm{x}$ & 13,730 & 12,560 & $9,225^{* * *}$ & $6,684^{*}$ \\
\hline & $(11,290-16,700)$ & $(10,100-15,610)$ & $1-11,270)$ & $(4,476-9,982)$ \\
\hline $\mathrm{C}_{\max }(\mathrm{ng} / \mathrm{mL})$ & $\begin{array}{l}1,471 \\
(1,222-1,770)\end{array}$ & $\begin{array}{l}1,350 \\
(1,109-1,645)\end{array}$ & $\begin{array}{l}1,134^{* * *} \\
(946-1,361)\end{array}$ & $1,031(831-1,278)$ \\
\hline 4- & $4-$ & $4-$ & 4- & $4-$ \\
\hline \multicolumn{5}{|c|}{$\begin{array}{l}\text { AcetylaminoantipyriAeetylaminoantipyriAeetylaminoantipyriAeetylaminoantipyriAeetylaminoantipyrine } \\
\begin{array}{lllll}\text { (4-AAA) } & \text { (4-AAA) } & \text { (4-AAA) } & \text { (4-AAA) } & \text { (4-AAA) }\end{array}\end{array}$} \\
\hline $\mathrm{AUC}_{12 \mathrm{~h}}(\mu \mathrm{g} / \mathrm{L} \mathrm{x}$ & 11,410 & 10,460 & $7,660^{* * *}$ & $5,298^{*}$ \\
\hline h) & $(7,742-16,830)$ & $(6,990-15,640)$ & $(5,031-11,660)$ & $(3,627-7,740)$ \\
\hline $\mathrm{C}_{\max }(\mathrm{ng} / \mathrm{mL})$ & $1,412(989-2,018)$ & $\begin{array}{l}1,412 \\
(1,012-1,969)\end{array}$ & $1,358(968-1,904)$ & $\begin{array}{l}1,322 \\
(1,003-1,742)\end{array}$ \\
\hline
\end{tabular}


Figure 1

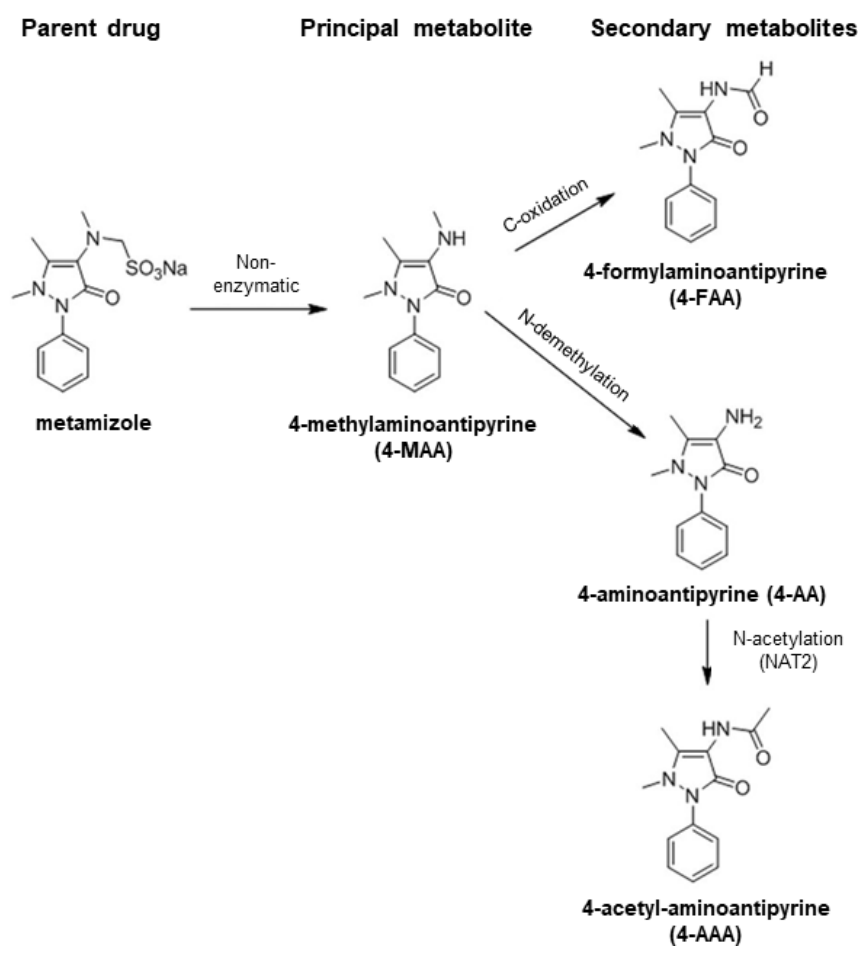

Figure 2
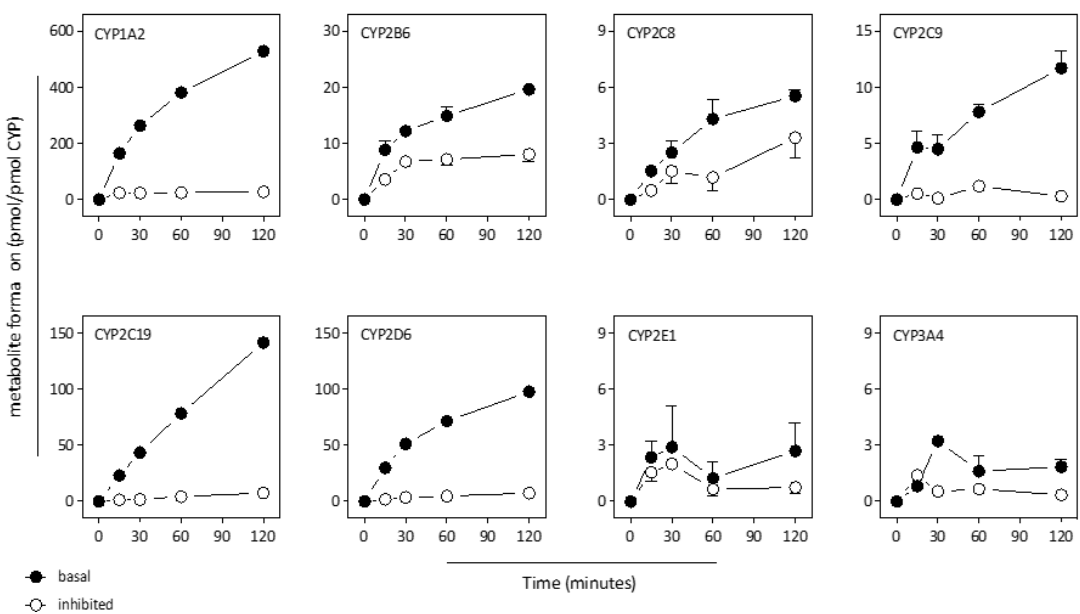

O inhibited 
Figure 3
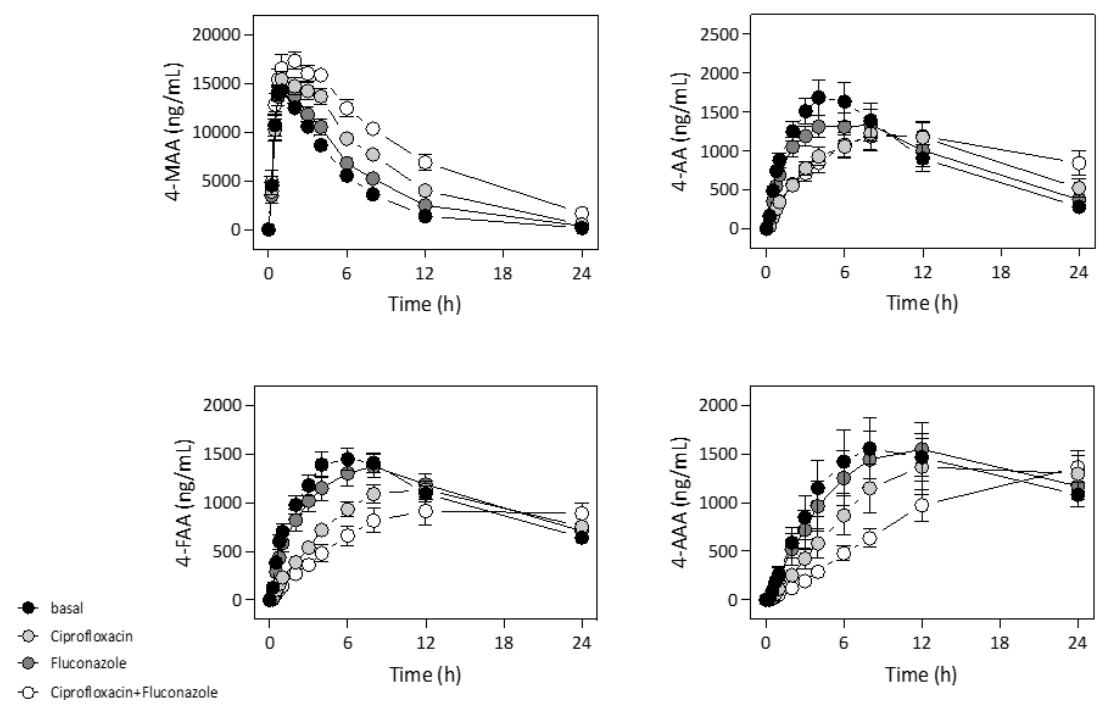

Figure 4
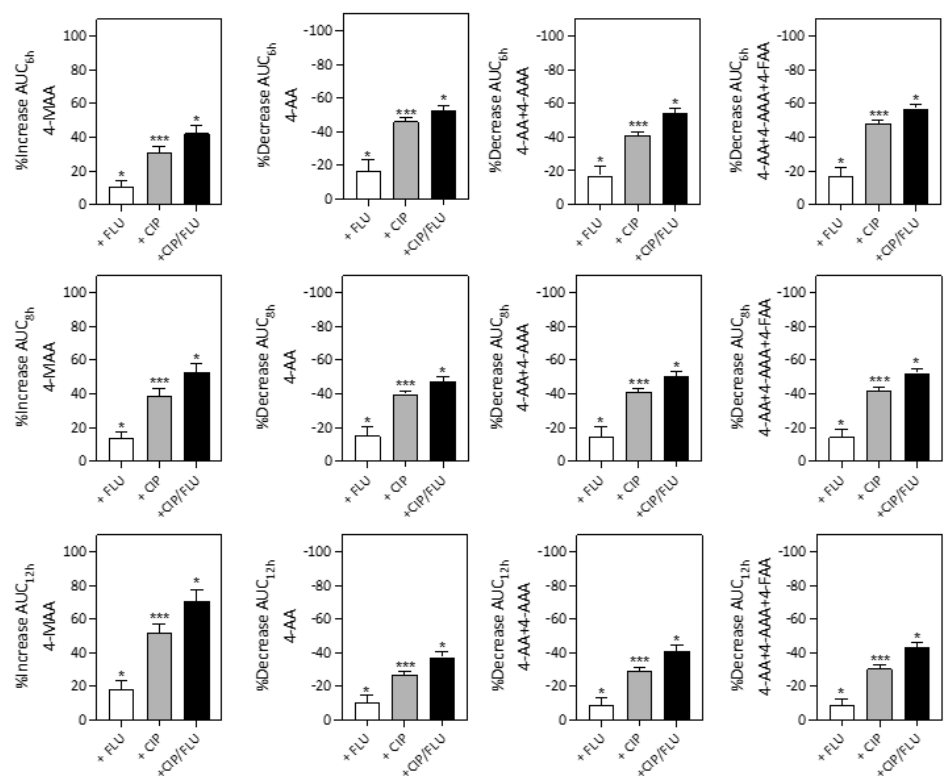
Figure 5
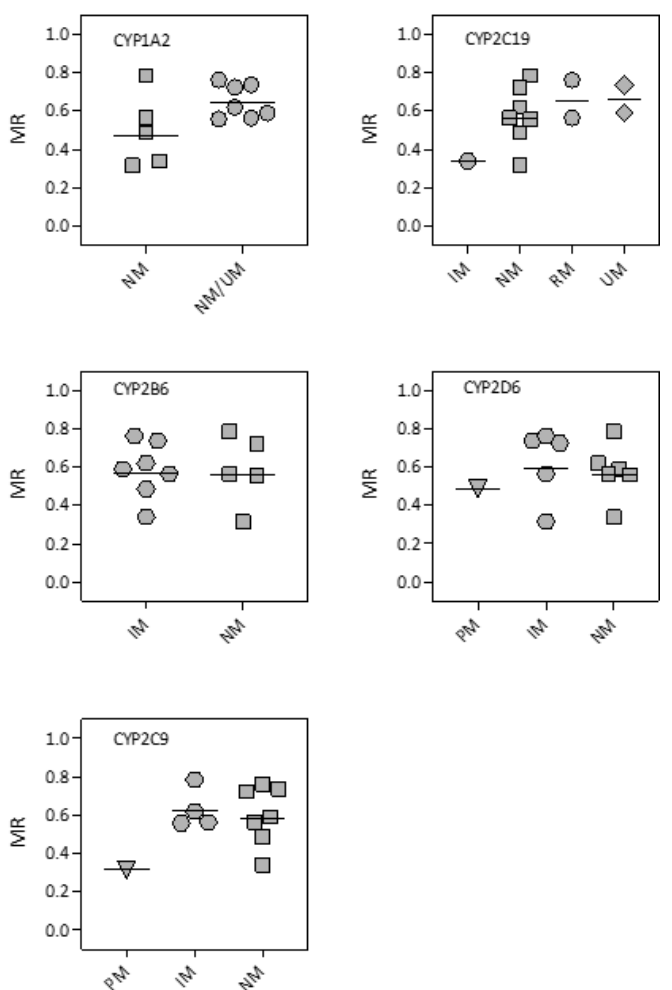